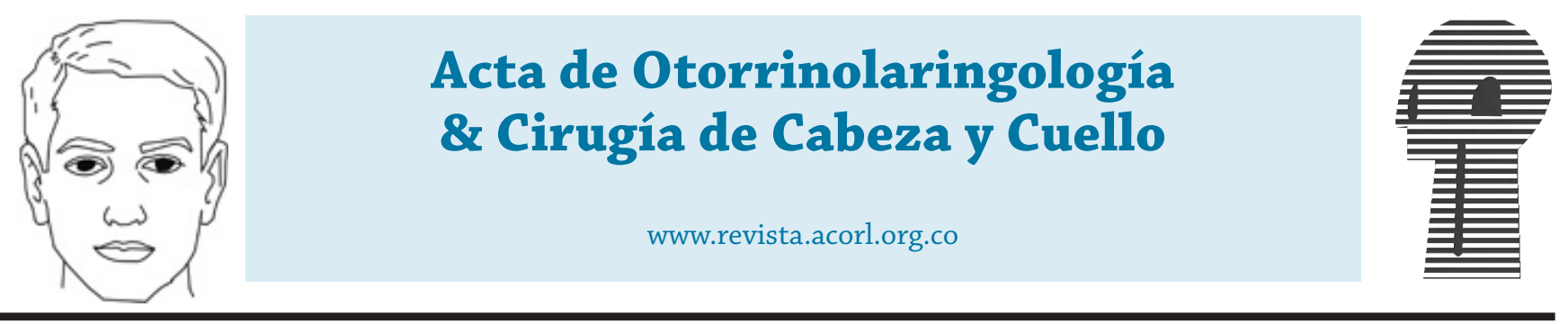

\title{
Quiste dermoide en base de cráneo con trayecto fistuloso a punta nasal en un paciente pediátrico: reporte de caso
}

\section{Skull base dermoid cyst fistulizing to the nasal tip in a pediatric patient: case report}

\author{
Martín Pinzón*, Catalina Jaramillo-Moncayo**, Manuela Orozco-Naranjo***. \\ * Instructor asociado, Departamento de Otorrinolaringología, Fundación Universitaria de Ciencias de la Salud, Hospital de San \\ José de Bogotá. Bogotá, Colombia. \\ ** Residente de Otorrinolaringología, Fundación Universitaria de Ciencias de la Salud, Hospital de San José de Bogotá. Bogotá, \\ Colombia. \\ ***Otorrinolaringóloga, Fundación Universitaria de Ciencias de la Salud, Hospital de San José de Bogotá. Bogotá, Colombia.
}

Forma de citar: Pinzón M, Jaramillo-Moncayo C, Orozco-Naranjo M. Quiste dermoide en base de cráneo con trayecto fistuloso a punta nasal en un paciente pediátrico: reporte de caso. Acta otorrinolaringol. cir. cabeza cuello. 2021;49(4): 304-308 DOI.10.37076/acorl.v49i4.555

El video del caso participó en el Congreso Nacional de Otorrinolaringología - Bucaramanga 2020 en la categoría: Mejor audiovisual realizado por un especialista y/o por un residente, "Trabajo Luis Barreto Bruce".

\section{INFORMACIÓN DEL ARTÍCULO}

\section{Historia del artículo:}

Recibido: 28 de agosto de 2020

Evaluado: 6 de diciembre de 2021

Aceptado: 10 de diciembre de 2021

Palabras clave (DeCS):

quiste dermoide, cavidad nasal, base del cráneo, preescolar, anomalías congénitas, rinoplastia, informes de casos.

\section{RESUMEN}

El quiste dermoide es una lesión congénita poco frecuente, secundario a una regresión incompleta de tejido ectodérmico, y su incidencia es de 1/20 000-40 000 nacidos vivos. Debe realizarse una resección quirúrgica oportuna para prevenir el compromiso intracraneal y/o las complicaciones. El estudio imagenológico determina su extensión. Se han recomendado diferentes enfoques quirúrgicos, los cuales deben cumplir con los cuatro principios de Pollock. El siguiente estudio presenta el caso de un paciente pediátrico, de nueve meses de edad, con un quiste dermoide en la base del cráneo asociado con un trayecto fistuloso a punta nasal. La resonancia magnética nuclear (RMN) contrastada evidenció una masa quística en la región anterior a la crista galli comunicada con el tracto fistuloso a la punta nasal. El paciente fue llevado a cirugía para realizar la resección del quiste mediante rinoplastia externa combinada con abordaje endoscópico transnasal. Los resultados funcionales y estéticos fueron satisfactorios, sin recurrencia. Se evidenció una fístula de líquido cefalorraquídeo intraoperatoria corregida en el mismo tiempo quirúrgico. 


\section{ABSTRACT}

Key words (MeSH):

Dermoid Cyst; Nasal Cavity;

Skull Base; Child; Congenital

Abnormalities; Rhinoplasty; Case

Reports.
The dermoid cyst is a rare congenital lesion, secondary to an incomplete regression of ectodermal tissue. Its incidence is $1 / 20,000-40,000$ live births. Timely surgical resection must be performed to prevent intracranial involvement and/or complications. Imaging study determines its extension. Different surgical approaches have been recommended; all must adhere to Pollock's 4 principles. The following study presents the case of a pediatric patient, 9 months old, with a dermoid cyst at the skull base with a fistulous path to the nasal tip. Contrasted nuclear magnetic resonance revealed a cystic mass in the region anterior to the crista galli communicated with a fistulous tract at the nasal tip. The patient was taken to surgery for cyst resection by external rhinoplasty combined with a transnasal endoscopic approach with satisfactory functional and aesthetic results, without recurrence. A Cerebrospinal fluid fistula was evidenced, as an intraoperative complication, it was corrected in the same surgical time.

\section{Introducción}

Las lesiones congénitas de la línea medio facial son anomalías poco frecuentes que se presentan como quistes, senos o fístulas. Pueden estar confirmadas por apéndices dérmicos (pelo, glándulas sudoríparas y glándulas sebáceas) contenidos dentro de un epitelio escamoso estratificado (1). Los quistes nasales fueron reportados por primera vez por Cruvelhier en 1817 (2), y hasta la fecha representan la lesión de línea media facial más frecuente (3). Tienen una incidencia estimada reportada de 1:20 000-40 000 nacidos vivos (1), de los cuales el $12 \%$ se extienden a la base del cráneo y la lámina cribiforme (4). Pratt y colaboradores y Leperchey y colaboradores (5) propusieron como mecanismo fisiopatológico una regresión incompleta del tejido ectodérmico en la región prenasal o nasofrontal, la cual se extiende desde el foramen caecum hasta la punta nasal. Esta regresión se da normalmente durante el segundo mes de desarrollo intrauterino.

Generalmente, los quistes dermoides se detectan en la primera década de la vida (edad media de dos a tres años). En estos pacientes se presenta un tracto fistuloso asociado que puede generar una solución de continuidad de la piel en cualquier punto del dorso nasal, desde la base de la columela hasta la glabela; siendo la región nasal distal el sitio más común y generando una deformidad nasal secundaria, que suele ser el motivo de consulta más frecuente de estos pacientes (6). Los quistes son característicamente firmes, no comprimibles, no pulsátiles y no traslúcidos. Se considera patognomónica la presencia de anexos cutáneos en su interior. El diagnóstico diferencial incluye múltiples patologías como lipoma, glioma, encefalocele, neurofibroma, teratoma, quiste subcutáneo y osteoma $(5,6)$.

La mayoría de los quistes dermoides son de densidad líquida, visible por tomografía axial computarizada (TAC) o resonancia magnética nuclear (RMN) de senos paranasales (7). La TAC ayuda al diagnóstico y proporciona información sobre la invasión de estructuras óseas y la RMN ayuda a determinar la extensión intracraneal (7). Los hallazgos compatibles con extensión intracraneal incluyen crista galli bífida, ensanchamiento interorbitario, del foramen caecum y defectos de la lámina cribiforme (6). Los valores predictivos positivos de TAC y RMN son $85,7 \%$ y $100 \%$, respectivamente, con valores predictivos negativos del $50 \%(6,8)$.

La resección quirúrgica permanece como único tratamiento para los quistes dermoides nasales (9); sin el tratamiento quirúrgico oportuno, el quiste aumenta de tamaño progresivamente como parte de su historia natural y puede presentar complicaciones como infecciones a repetición y distorsión de cartílagos y/o huesos nasales con deformidad facial secundaria (10). Se han descrito diferentes técnicas quirúrgicas que se deben cumplir como requisito: una adecuada visualización quirúrgica, exposición, acceso para la resección completa, cierre del defecto óseo y reconstrucción cosmética final (11). Los enfoques tradicionales incluyen rinotomía lateral, facial o rinoplastia externa con o sin craneotomía. Recientemente, la cirugía endoscópica transnasal ha ganado importancia y preferencia sobre las otras técnicas quirúrgicas dado la baja tasa de complicaciones y menor comorbilidad asociada; en algunos casos, se puede indicar un tratamiento quirúrgico combinando los diferentes abordajes $(12,13)$.

El presente estudio reporta un caso interesante de un paciente pediátrico de nueve meses de edad con un quiste dermoide nasal asociado a tracto fistuloso desde la base del cráneo hasta la punta nasal. El diagnóstico se realizó con la clínica y estudio imagenológico (TAC y RMN). Se realizó la resección y extracción completa del quiste dermoide con una técnica quirúrgica combinada por rinoplastia externa y abordaje endoscópico transnasal.

\section{Reporte de caso}

Paciente masculino de nueve meses, quien se presenta al servicio de otorrinolaringología con un cuadro clínico desde el nacimiento consistente en una lesión nodular de la punta nasal, con crecimiento progresivo y deformidad nasal secundaria adquirida. La madre del paciente no refería síntomas 
adicionales. El paciente no tiene antecedentes familiares de importancia, incluyendo ausencia de antecedentes de malformaciones congénitas en la familia. En el examen físico se evidencia una lesión quística de $15 \mathrm{~mm}$ en el dorso nasal que compromete punta y suprapunta (Figura 1). El examen neurológico resultó normal.

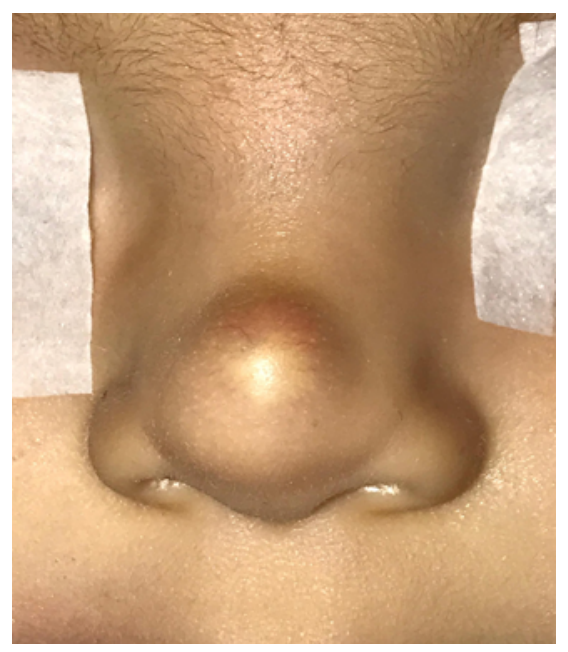

Figura 1. Vista frontal prequirúrgica del quiste dermoide nasal. Se muestra una lesión quística redondeada y bien delimitada que corresponde a un quiste dermoide que compromete una punta nasal. Imagen obtenida del paciente.

En la RMN de senos paranasales contrastada se evidenció una masa quística de forma ovalada en el espacio prenasal junto al foramen caecum, anterior a la crista galli, comunicándose a través de un tracto fistuloso con una segunda masa en el dorso y punta nasal; las dos masas se presentaban hiperintensas en T2 (Figura 2A). La TAC demostró una adecuada neumatización de los senos paranasales y permeabilidad de cavidad nasal. En el corte coronal se observa un ensanchamiento de la crista galli (Figura 2B).
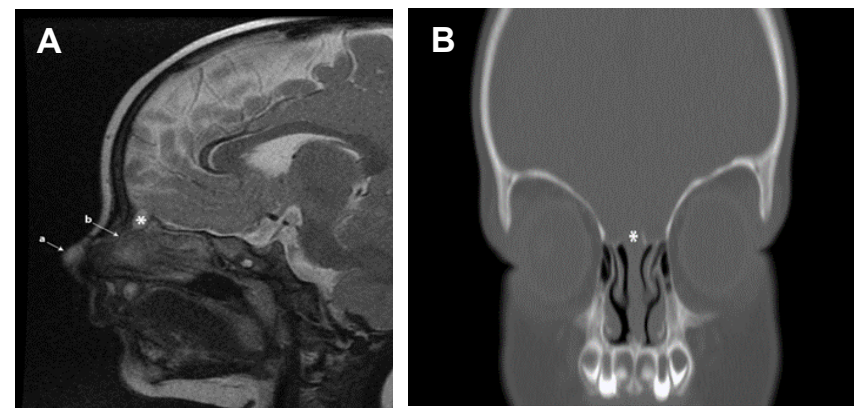

Figura 2. Estudio imagenológico prequirúrgico. A. Resonancia magnética nuclear de senos paranasales con contraste corte sagital. Masa quística en punta nasal (a) comunicándose a través del tracto fistuloso (b) con una segunda masa a nivel del foramen caecum (*). B. Tomografía axial computarizada corte coronal.

El paciente fue llevado a resección de dos quistes dermoides (base de cráneo y punta nasal) mediante un abordaje nasal abierto (rinoplastia externa) combinado con un abordaje endoscópico transnasal. Bajo anestesia general inicialmente se obtuvo un injerto graso del área supraumbilical, el cual se utilizó posteriormente para la reconstrucción del dorso nasal. Se realizaron incisiones medias columelares y marginales para la disección y elevación de la piel del dorso nasal seguido de la exposición del septo nasal al separar las cruras mediales. Se identificó el quiste dermoide de la punta nasal junto con el tracto fistuloso y remanentes ectodérmicos (Figura 3). Se realizó una disección de los túneles submucopericóndricos del septo nasal derecho y se continuó el abordaje bajo visión endoscópica siguiendo la disección del tracto fistuloso; por último, se alcanzó el quiste dermoide en la region prenasal a través de un foramen caecum patente, anterior a la crista galli.

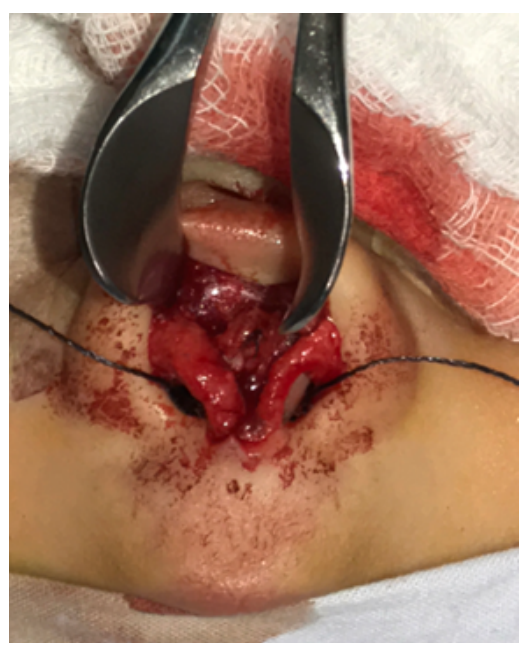

Figura 3. Vista frontal intraquirúrgica del quiste dermoide nasal. Se muestra la elevación del colgajo bipediculado, la identificación del quiste entre las curas mediales junto con el tracto fistuloso y los remanentes ectodérmicos. Imagen obtenida del paciente. [Nota: confirmar si la corrección es adecuada]

Tanto el contenido de los quistes como los revestimientos epiteliales se extrajeron gradualmente. No hubo extensión intradural, sin embargo, posterior a la resección completa del quiste hubo una fístula de líquido cefalorraquídeo (LCR) que fue reparada y revestida con sellante de fibrina y agente hemostático absorbible. Con la resección del quiste de la punta nasal se observó una solución de continuidad lineal en la piel del dorso nasal y se corrigió el espacio muerto del dorso nasal con el injerto de grasa obtenido previamente. El procedimiento quirúrgico finalizó con el cierre de heridas en la piel (dorso y columela) (Figura 4) y se posicionó un vendaje blando sobre el dorso nasal por una semana.

El seguimiento fue satisfactorio, sin complicaciones; el paciente fue dado de alta el mismo día. En el control posoperatorio, una semana después del procedimiento quirúrgico, el paciente presentaba un edema leve en la punta nasal sin otros síntomas asociados. En el seguimiento a un año se evidenció la resolución completa del edema en la punta nasal, sin signos de recurrencia, sin obstrucción nasal, ausencia de rinoliquia y con un resultado estético satisfactorio. 


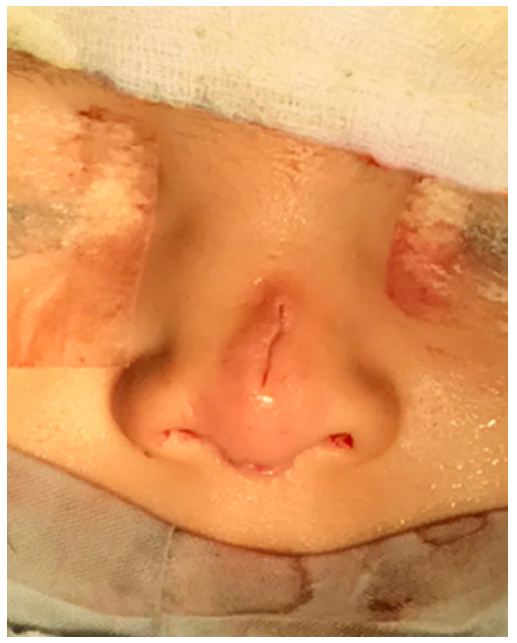

Figura 4. Vista frontal de la incisión en la punta nasal. Se muestra el resultado posoperatorio inmediato del cierre del abordaje suprapunta y el dorso nasal. Imagen obtenida del paciente.

\section{Discusión}

La presentación clínica de masas en punta nasal, como en nuestro caso, oscilan entre $4 \%$ a $12 \%$ (1). El diagnóstico inicial se puede realizar fácilmente en función de los hallazgos clínicos (4). Los quistes dermoides se asocian frecuentemente con múltiples anomalías; las series de casos reportan una prevalencia de esta asociación que va del $6 \%$ al $41 \%$ (3), presentándose con mayor frecuencia en asociación con una extensión intracraneal (56\%) en comparación con una presentación aislada del quiste dermoide nasal (33 \%) (3). Nuestro caso tenía un quiste dermoide aislado sin extensión intracraneal evidenciada durante el procedimiento quirúrgico. El estudio imagenológico, contrario a los hallazgos intraquirúrgicos, generaban una alta sospecha de compromiso intracraneal por los hallazgos en la TAC de senos paranasales con ensanchamiento de la crista galli (6, 10). Según Pensler y colaboradores (12), la presencia de ensanchamiento de un septo nasal bífido, una crista galli bífida, ensanchamiento interorbitario y defectos de la lámina cribiforme sugieren extensión intracraneal; frente a estos hallazgos en la TAC, se debe complementar el estudio con una RMN de los senos paranasales $(3,12)$. El estudio imagenológico prequirúrgico, por lo tanto, es crucial para confirmar el diagnóstico y excluir otras patologías, ya que ayuda a determinar la extensión. Se debe realizar un protocolo de RMN con cortes finos multiplanar (2-3 mm), T1 de alta resolución, $\mathrm{T} 2$ con supresión grasa e incluir secuencia con restricción a la difusión. La TAC debe tener también un protocolo que incluya cortes finos para proporcionar información detallada sobre la anatomía ósea (1).

La tasa de recurrencia del quiste dermoide nasal, al ser manejado mediante un abordaje nasal abierto, es del 5,5 $\%$ según lo reportado en la literatura. En nuestro paciente se realizó un abordaje combinado con rinoplastia externa, lo que permitió una amplia exposición del sitio quirúrgico, de la lesión, un mejor control sobre las estructuras vecinas y osteotomías, en caso de ser necesarias; en nuestro caso permitió una disección cuidadosa del tracto fistuloso hasta la base del cráneo, cumpliendo así los 4 principios de Pollock (11). La técnica quirúrgica fue complementada con un abordaje endoscópico transnasal ante a la sospecha de una extensión intracraneal. La fístula de LCR es una complicación poco frecuente, sin embargo, se ha documentado debido a la probabilidad de lesión iatrogénica de la lámina cribiforme $(13,14)$. El abordaje combinado con cirugía endoscópica transnasal y rinoplastia externa permite identificarla de forma oportuna y corregirla en el mismo tiempo quirúrgico sin mayores complicaciones adicionales, como en nuestro paciente $(10,15)$.

\section{Conclusión}

Los quistes dermoides nasales son una lesión congénita de la línea media poco frecuentes. La población pediátrica con diagnóstico de quiste dermoide nasal, el diagnóstico diferencial y determinar la extensión intracraneal son cruciales. Los pacientes deben ser estudiados mediante TAC y RMN en los casos en los que la TAC sola no es concluyente. El planeamiento quirúrgico se basa en una exposición adecuada que permite una disección y una resección completas de la lesión, así como una reconstrucción de la base del cráneo, en caso de ser necesario, y resultados estéticos satisfactorios. Actualmente un abordaje combinado con cirugía endoscópica transnasal y rinoplastia externa es el tratamiento de elección.

\section{Consideraciones éticas}

Se obtuvo consentimiento informado por parte de los padres del paciente de acuerdo con los principios éticos contenidos en la Declaración de Helsinki.

\section{Conflicto de interés}

Los autores declaramos haber revisado y aprobado el manuscrito sometido a su consideración y aprobamos su publicación. Adicionalmente, los autores declaramos no tener ningún conflicto de interés.

\section{Financiación}

Esta publicación no tuvo financiación.

\section{REFERENCIAS}

1. Herrington H, Adil E, Moritz E, Robson C, Perez-Atayde A, Proctor M, et al. Update on current evaluation and management of pediatric nasal dermoid. Laryngoscope. 2016;126(9):215160. doi: 10.1002/lary.25860

2. Pratt L. Midline cyst of the nasal dorsum: Embryologic origin and treatment. Laryngoscope. 1965;75:965-80. doi: 10.1288/00005537-196506000-0010 
3. Denoyelle F, Ducroz V, Roger G, Garabedian EN. Nasal dermoid sinus cysts in children. Laryngoscope. 1997;107(6):795-800. doi: 10.1097/00005537-199706000-00014

4. Makhdoom N, Abo El Ezz TA, Abdel-Haleem M. Management of midline nasal dermoid lesions in children by external rhinoplasty. J Taibah Univ Med Sci. 2017;12(4):324-28. doi: 10.1016/j.jtumed.2017.02.003

5. Leperchey F, Cotin G, Bodard M, Garabedian N, Klap P. Deux modalités dans le processus embryogénique des kystes et fistules fronto-nasaux [2 modalities in the embryogenic process of frontonasal cysts and fistulae]. Ann Otolaryngol Chir Cervicofac. 1983;100(4):247-54.

6. Winterton RIS, Wilks DJ, Chumas PD, Russell JL, Liddington MI. Surgical correction of midline nasal dermoid sinus cysts. J Caniofacial Surg. 2010;21(2):295-300. doi: 10.1097/ SCS.0b013e3181cf5f44

7. Kadom N, Sze RW. Radiological reasoning: Pediatric midline nasofrontal mass. Am J Roentgenol. 2010;194(3 SUPPL.):10-13. doi: 10.2214/AJR.07.7126

8. Ortlip T, Ambro BT, Pereira KD. Midline approach to pediatric nasofrontal dermoid cysts. JAMA Otolaryngol Head Neck Surg. 2015;141(2):174-77. doi:10.1001/jamaoto.2014.3185

9. Cheng J, Kazahaya K. Management of pediatric nasal dermoids with intracranial extension by direct excision. Otolaryngol Head Neck Surg. 2013;148(4):694-96. doi: $10.1177 / 0194599812474424$
10. Bilkay U, Gundogan H, Ozek C, Tokat C, Gurler T, Songur E, et al. Nasal dermoid sinus cysts and the role of open rhinoplasty. Ann Plast Surg. 2001;47(1):8-14. doi: 10.1097/00000637200107000-00002

11. Pollock RA. Surgical approaches to the nasal dermoid cyst. Ann Plast Surg. 1983;10(6):498-01. doi: 10.1097/00000637198306000-00012

12. Re M, Tarchini P, Macrì G, Pasquini E. Endonasal endoscopic approach for intracranial nasal dermoid sinus cysts in children. Int J Pediatr Otorhinolaryngol. 2012;76(8):1217-22. doi: 10.1016/j.ijporl.2012.05.004

13. Livingstone DM, Brookes J, Yunker WK. Endoscope-assisted nasal dermoid excision with an open rhinoplasty approach. Int J Pediatr Otorhinolaryngol. 2018;109:101-3. doi: 10.1016/j. ijporl.2018.03.035

14. Soni RS, Choudhry OJ, Liu JK, Eloy JA. Postoperative cerebrospinal fluid leak after septoplasty: A potential complication of occult anterior skull base encephalocele. Allergy Rhinol (Providence). 2013;4(1):e41-4. doi: 10.2500/ ar.2013.4.0043

15. Kryzanski JT, Annino DJ, Gopal H, Heilman CB. Low complication rates of cranial and craniofacial approaches to midline anterior skull base lesions. Skull Base. 2008;18(4):22942. doi: 10.1055/s-2007-1003924. 